\title{
Approximating connectivity augmentation problems
}

\author{
Zeev Nutov \\ The Open University of Israel
}

Let $G=(V, E)$ be an undirected graph and let $S \subseteq V$. The $S$-connectivity $\lambda_{G}^{S}(u, v)$ of a node pair $(u, v)$ in $G$ is the maximum number of $u v$-paths that no two of them have an edge or a node in $S-\{u, v\}$ in common. The corresponding Connectivity Augmentation (CA) problem is: given a graph $G=(V, E)$, a node subset $S \subseteq V$, and a nonnegative integer requirement function $r(u, v)$ on $V \times V$, add a minimum size set $F$ of new edges to $G$ so that $\lambda_{G+F}^{S}(u, v) \geq r(u, v)$ for all $(u, v) \in$ $V \times V$. Three extensively studied particular cases are: the Edge-CA $(S=\emptyset)$, the Node-CA $(S=V)$, and the Element-CA $(r(u, v)=0$ whenever $u \in S$ or $v \in S)$. A polynomial algorithm for Edge-CA was developed by Frank. In this paper we consider the Element-CA and the Node-CA, that are NP-hard even for $r(u, v) \in\{0,2\}$. The best known ratios for these problems were: 2 for ElementCA and $O\left(r_{\max } \cdot \ln n\right)$ for Node-CA, where $r_{\max }=\max _{u, v \in V} r(u, v)$ and $n=|V|$. Our main result is a $7 / 4$-approximation algorithm for the Element-CA, improving the previously best known 2-approximation. For Element-CA with $r(u, v) \in\{0,1,2\}$ we give a 3/2-approximation algorithm. These approximation ratios are based on a new splitting-off theorem, which implies an improved lower bound on the number of edges needed to cover a skew-supermodular set function. For NodeCA we establish the following approximation threshold: Node-CA with $r(u, v) \in\{0, k\}$ cannot be approximated within $O\left(2^{\log ^{1-\varepsilon} n}\right)$ for any fixed $\varepsilon>0$, unless $\operatorname{NP} \subseteq \operatorname{DTIME}\left(n^{\operatorname{polylog}(n)}\right)$.

Categories and Subject Descriptors: F.2.2 [Nonnumerical Algorithms and Problems]: Computations on discrete structures; G.2.2 [Discrete Mathematics]: Graph Algorithms

General Terms: Approximation Algorithms, Hardness of Approximation

Additional Key Words and Phrases: connectivity augmentation, element-connectivity, nodeconnectivity

\section{INTRODUCTION}

\subsection{Problem statement}

Let $G=(V, E)$ be a graph and let $S \subseteq V$. The $S$-connectivity $\lambda_{G}^{S}(u, v)$ of $(u, v)$ in $G$ is the maximum number of $u v$-paths that no two of them have an edge or a node in $S-\{u, v\}$ in common. Unless stated otherwise, all graphs are assumed to be undirected. We consider the following problem:

Connectivity Augmentation (CA):

Instance: A graph $G=(V, E), S \subseteq V$, and a requirement function $r(u, v)$ on $V \times V$.

A preliminary version of this paper appeared in Proc. SODA 2005, pp. 176-185.

Authors' address: Zeev Nutov, Department of Computer Science, The Open University of Israel, Raanana, Israel. E-mail: nutov@openu.ac.il.

Permission to make digital/hard copy of all or part of this material without fee for personal or classroom use provided that the copies are not made or distributed for profit or commercial advantage, the ACM copyright/server notice, the title of the publication, and its date appear, and notice is given that copying is by permission of the ACM, Inc. To copy otherwise, to republish, to post on servers, or to redistribute to lists requires prior specific permission and/or a fee.

(c) $20 \mathrm{YY}$ ACM 0000-0000/20YY/0000-0001 $\$ 5.00$ 
Objective: Find a minimum size edge set $F$ so that $\lambda_{G+F}^{S}(u, v) \geq r(u, v)$ for all $(u, v) \in V \times V$.

Three extensively studied particular cases are: the Edge-CA $(S=\emptyset)$, the Node-CA $(S=V)$, and the Element-CA $(r(u, v)=0$ whenever $u \in S$ or $v \in S)$. A particular case of the Element-CA is the Hypergraph-Edge-CA where the goal is to augment a given hypergraph by edges (hyperedges of size two) to meet given pairwise (hyper)edge connectivity requirements, see Proposition 4.4. CA is a particular case of the Generalized Steiner Network problem, where we are given a complete graph with costs on the edges, $S \subseteq V$, and connectivity requirements $r(u, v)$, and the goal is to find a minimum-cost subgraph that satisfies the connectivity requirements. CA is the case of 0,1-costs, when the edges of $G$ have cost 0 , and any other edge is allowed by cost 1. See [Kortsarz and Nutov 2007] for classification of Generalized Steiner Network problems with respect to costs and requirements.

While the Edge-CA was shown to be polynomially solvable in [Frank 1992], the Hypergraph-Edge-CA and the Node-CA are NP-hard even when the input graph $G$ is connected and $r(u, v) \in\{0,2\}$, see [Cosh et al. 2003] and [Nagamochi and Ishii 2003], respectively; the NP-hardness proofs in [Cosh et al. 2003] and [Nagamochi and Ishii 2003] are essentially identical. The best known ratios for CA problems are as follows. [Kortsarz and Nutov 2008] established approximation ratios $O(\ln n)$ for $S \neq V$ and $O\left(r_{\max } \cdot \ln n\right)$ for Node-CA (the case $S=V$ ), where $r_{\max }=\max _{u, v \in V} r(u, v)$ and $n=|V|$; this result is valid for both directed and undirected graphs. In fact, for large values of $r_{\max }$ directed and undirected NodeCA problems were recently shown to be equivalent w.r.t. approximation in [Lando and Nutov 2009]. The Element-CA admits a 2-approximation algorithm via an extension of the iterative LP-rounding method of [Jain 2001], which applies also to the min-cost version of the problem, see [Fleischer et al. 2006] and [Cheriyan et al. 2006]. [Nagamochi and Ishii 2003] gave a combinatorial 3/2-approximation algorithm for Node-CA with $r(u, v) \in\{0,2\}$ when the input graph $G$ is connected; this algorithm applies to Element-CA as well.

Here is some additional notation used in the paper. An edge between $u, v$ is denoted by $u v$. A $u v$-path is a (simple) path with endnodes $u, v$. For an arbitrary two sets $A, B$ of nodes and edges (or graphs) $A-B$ is the set (or graph) obtained by deleting $B$ from $A$, where deletion of a node implies also deletion of all the edges incident to it; similarly, $A+B$ is the set (graph) obtained by adding $B$ to $A$. Let $H$ be a graph or an edge set on node set $V$. For disjoint $X, Y \subseteq V$ we denote by $\delta_{H}(X, Y)$ the set $\{u v \in H: u \in X, v \in Y\}$ of the edges in $H$ from $X$ to $Y$ and $d_{H}(X, Y)=\left|\delta_{H}(X, Y)\right|, \delta_{H}(X)=\delta_{H}(X, V-X)$, and $d_{H}(X)=\left|\delta_{H}(X)\right|$. Let $\kappa_{H}(u, v)=\lambda_{H}^{V}(u, v)$ be the node-connectivity between $u, v$. Let opt denote the optimal solution value of a problem instance at hand. Let $Z_{+}$denote the set of non-negative integers. For a function $g$ on $V$ and $X \subseteq V$ let $g(X)=\sum_{v \in X} g(v)$.

\subsection{Our results}

Recall that Element-CA is NP-hard even when $r(u, v) \in\{0,2\}$ and $G$ is connected [Cosh et al. 2003], and that in this case the problem admits a 3/2-approximation algorithm [Nagamochi and Ishii 2003]. An approximation ratio better than 2 was not known for other versions of the problem. We prove: 
THEOREM 1.1. Element-CA admits a 7/4-approximation algorithm for arbitrary requirements, and a 3/2-approximation algorithm if $r(u, v) \in\{0,1,2\}$. The same holds for Hypergraph Edge-CA.

Theorem 1.1 is based on a new splitting-off theorem, which also implies an improved lower bound on the number of edges needed to cover a skew-supermodular set function. We need some definitions to present this result.

DeFinition 1.1. A set-function $p: 2^{V} \rightarrow Z_{+}$is symmetric if $p(X)=p(V-X)$ for all $X \subseteq V$, and $p$ is skew-supermodular if $p(\emptyset)=0$ and for any $X, Y \subseteq V$ with $p(X), p(Y)>0$ at least one of the following holds:

$$
\begin{aligned}
& p(X)+p(Y) \leq p(X \cap Y)+p(X \cup Y) \\
& p(X)+p(Y) \leq p(X-Y)+p(Y-X)
\end{aligned}
$$

If (1) always holds for any $X, Y \subseteq V$ with $p(X), p(Y)>0$ then $p$ is supermodular.

DeFinition 1.2. An edge set $F$ on $V$ covers a set function $p$, or $F$ is a p-cover, if $d_{F}(X) \geq p(X)$ for all $X \subseteq V$. A function $g: V \rightarrow Z_{+}$is a $p$-transversal if $g(X) \equiv \sum\{g(v): v \in X\} \geq p(X)$ for all $X \subseteq V$. Let $T_{g}=\{v \in V: g(v) \geq 1\}$. A p-transversal $g$ is a minimal $p$-transversal if for any $v \in T_{g}$ reducing $g(v)$ by 1 results in a function that is not a p-transversal.

CA problems can be casted as the following problem, c.f. [Frank and Jordán 1995; Benczúr and Frank 1999; Frank 1992; Jain 2001; Fleischer et al. 2006; Cheriyan et al. 2006; Kortsarz and Nutov 2007].

\section{Set-Function Edge-Cover}

Instance: A set-function $p$ on a groundset $V$.

Objective: Find a minimum size $p$-cover.

Let $\operatorname{opt}(p)$ be the minimum size of a $p$-cover. As $g(v)=d_{F}(X)$ is a $p$-transversal for any $p$-cover $F$, opt $(p) \geq g(V) / 2$ for any $p$-transversal $g$ with $g(V)$ minimum. For a skew-supermodular $p$, it is known that any minimal $p$-transversal $g$ minimizes $g(V)$, see Lemma 3.2. Thus a natural approach to compute a small $p$-cover is: repeatedly choose an edge $u v$ with $u, v \in T_{g}$, so that updating $p$ and reducing $g(u)$ and $g(v)$ by 1 , keeps $g$ being a $p$-transversal. This approach works for some special cases, but in general such an edge $u v$ may not exist.

More formally, given $u, v \in T_{g}$ define $p^{u v}$ and $g^{u v}$ as follows:

$$
\begin{gathered}
p^{u v}(X)=\max \{p(X)-1,0\} \text { if }|X \cap\{u, v\}|=1 \text { and } p^{u v}(X)=p(X) \text { otherwise; } \\
g^{u v}(x)=g(x)-1 \text { if } x=u \text { or if } x=v \text { and } g^{u v}(x)=g(x) \text { otherwise. }
\end{gathered}
$$

It is easy to see that if $p$ is (symmetric) skew-supermodular, so is $p^{u v}$, e.g. see [Jain 2001]. However, $g^{u v}$ may not be a $p^{u v}$-transversal if $g$ is. We say that a pair $u, v \in T_{g}$ is $(p, g)$-legal if $g^{u v}$ is a $p^{u v}$-transversal; then replacing $p, g$ by $p^{u v}, g^{u v}$ is the splitting-off operation at $u, v$. Intuitively, splitting-off is an attempt to add the edge $u v$ to a partial solution, and to consider the residual problem of covering $p^{u v}$ with the residual lower bound $\left\lceil g^{u v}(V) / 2\right\rceil=\lceil g(V) / 2\rceil-1$.

We consider the simplest algorithm which repeatedly splits-off legal pairs as long as such exist, and then adds to the partial solution any inclusion minimal solution 
of the residual instance. We will characterize those pairs $p, g$ for which no $(p, g)$ legal pair exists, and obtain a new lower bound on opt $(p)$ for a skew supermodular set-function $p$.

It would be convenient to describe our results in terms of the projection function $p_{g}$ of $p$ on $T_{g}$ defined by $p_{g}(T)=\max \left\{p(X): X \subseteq V, X \cap T_{g}=T\right\}$ for all $T \subseteq T_{g}$. It follows from the definition of $p_{g}$ that any $p_{g}$-cover is also a $p$-cover. The following statement essentially says that w.l.o.g. we may consider edge-covering $p_{g}$ instead of $p$. It was implicitly proved in [Benczúr and Frank 1999], and we will prove a generalization of it in Section 4.1.

LEMMA 1.2. Let $p$ be symmetric skew-supermodular and let $g$ be a minimal $p$ transversal. Then $p_{g}$ is symmetric skew-supermodular, any $p_{g}$-cover is a $p$-cover, and $\operatorname{opt}(p)=\operatorname{opt}\left(p_{g}\right)$.

THEOREM 1.3. Let $p$ be symmetric skew-supermodular, let $g$ be a minimal $p$ transversal, and suppose that no $(p, g)$-legal pair exists. Then $p$ and $g$ are 0,1 valued, $\left|T_{g}\right| \geq 3$, and $p_{g}(T)=1$ for all $T \subset T_{g}$ with $|T| \in\{1,2\}$. Furthermore, $\operatorname{opt}(p)=\operatorname{opt}\left(p_{g}\right) \geq\left\lceil 2\left|T_{g}\right| / 3\right\rceil$.

Based on Theorem 1.3 we give an approximation algorithm for Set-Function EdgeCover with skew-supermodular $p$, but its polynomial implementation requires that certain queries related to $p$ can be answered in polynomial time. For an edge set $F$ and node set $X$ let $\delta_{F}(X)$ denote the set of edges in $F$ with exactly one endnode in $X$. Following [Benczúr and Frank 1999], we assume that for any edge set $F$ with integral weights $\{w(e): e \in F\}(w(e)$ is the multiplicity of the edge $e)$ and any function $g$ on $V$ we have a polynomial time oracle for

$$
\min _{X \subseteq V}\left(g(X)+w\left(\delta_{F}(X)\right)-p(X)\right)
$$

As was pointed in [Benczúr and Frank 1999], for a supermodular $p$ (3) can be realized in polynomial time even if $p$ is given by an evaluation oracle. Such an extension is not known for skew-supermodular functions. But in applications discussed in this paper, (3) can be realized in polynomial time via max-flows.

TheOREM 1.4. Assuming a polynomial time oracle for (3) is available, SetFunction Edge-Cover with skew-supermodular $p$ admits a 7/4-approximation algorithm. Furthermore, the problem is APX-hard even for 0,1-valued set functions for which no legal pair exists.

The APX-hardness reduction in Theorem 1.4 does not seem to be straightforwardly applicable to $p$-cover problems arising from augmentation problems. For example, the Edge-CA is in $\mathrm{P}$.

In [Benczúr and Frank 1999] was developed a polynomial time algorithm that computes an optimal edge-cover of a symmetric supermodular $p$, under the assumption that a polynomial time oracle for (3) is available. They used the following lower bound. A subpartition $\mathcal{F}$ of $V$ is $p$-full if $p\left(\cup_{X \in \mathcal{F}^{\prime}} X\right) \geq 1$ for every $\emptyset \subset \mathcal{F}^{\prime} \subset \mathcal{F}$. It is easy to see that any $p$-cover $F$ must induce a connected graph on the parts of a $p$-full subpartition $\mathcal{F}$, hence $|F| \geq|\mathcal{F}|-1$. Let $\operatorname{dim}(p)$ denote the maximum cardinality of a $p$-full subpartition. Let $g$ be a $p$-transversal with $g(V)$ minimum. From the discussion above it follows that opt $(p) \geq \max \{\lceil g(V) / 2\rceil, \operatorname{dim}(p)-1\}$ for 
any set-function $p$. [Benczúr and Frank 1999] showed that for a symmetric supermodular $p$ an (optimal) $p$-cover of size $\max \{\lceil g(V) / 2\rceil, \operatorname{dim}(p)-1\}$ can be computed in polynomial time. Later, [Szigeti 1999] showed that for an even valued symmetric skew-supermodular $p$ the bound $\lceil g(V) / 2\rceil$ is achievable.

However, for an arbitrary skew-supermodular $p$ the lower bound of [Benczúr and Frank 1999] is not sufficient to get an approximation $2-\varepsilon$ for some fixed $\varepsilon$ even for 0,1 -valued $p$. To see this let $|V|=k \cdot \ell$, let $A_{1}, \ldots, A_{\ell}$ be a partition of $V$ into $\ell$ sets of size $k$ each, and let $\mathcal{F}=\left\{X: \emptyset \neq X \subset A_{i}\right.$ for some $\left.i\right\}$. Set $p(X)=1$ if $X \in \mathcal{F}$ or if $V-X \in \mathcal{F}$. It is easy to verify that: $p$ is symmetric skew-supermodular, $g(v)=1$ for all $v \in V$ is a minimal $p$-transversal so $g(V)=|V|=k \cdot \ell$, and $\operatorname{opt}(p)=|V|-\ell=\ell(k-1)$ (an optimal solution is a union of trees on each $\left.A_{i}\right)$. On the other hand, $\operatorname{dim}(p)=k$ (a maximum $p$-full subpartition consists of all singletons from some $\left.A_{i}\right)$. The ratio between opt $(p)$ and the lower bound $\max \{g(V) / 2, \operatorname{dim}(p)-1\}=k \cdot \ell / 2$ is $2(k-1) / k$, which approaches 2 if $k$ is large.

Our 3/2-approximation algorithm for Element-CA with $r(u, v) \in\{0,1,2\}$ is based on a better lower bound than the one in Theorem 1.3. It is easy to see that if $p$ is 0,1 valued, then any inclusion minimal $p$-cover is a forest. Assume that no $(p, g)$-legal pair exists. Then any tree on $T_{g}$ is a $p$-cover, since $p$ is 0,1 -valued, by Theorem 1.3. The lower bound in Theorem 1.3 gives a $3 / 2$-approximation for instances without legal pairs, by just taking any tree on $T_{g}$. We prove that if $r(u, v) \in\{0,1,2\}$, then for the corresponding set-function $p$ the problem can be solved in polynomial time on instances without legal pairs. Specifically, we show that there exists a partition $\mathcal{T}$ of $T_{g}$ so that $p_{g}(X)=1$ for $X \subset T_{g}$ if, and only if, $X$ divides some part of $\mathcal{T}$; a set $X$ divides a set $T$ if $X \cap T, T-X \neq \emptyset$. This implies that any inclusion minimal or optimal $p_{g}$-cover is a union of trees on the parts of $\mathcal{T}$.

Our last result is for the Node-CA. [Kortsarz et al. 2004] established an approximation threshold for the problem of finding a min-size spanning subgraph $G$ of a given graph $H$ so that $\kappa_{G}(u, v) \geq r(u, v)$ for all $u, v \in V$ (this is the case of $\{1, \infty\}$ costs) even when $r(u, v) \in\{0, k\}$. By extending the construction of [Kortsarz et al. 2004], we will show a similar hardness result for the (usually easier) augmentation version (the $\{0,1\}$ costs case), and prove:

TheOREM 1.5. Node-CA with $r(u, v) \in\{0, k\}$ cannot be approximated within $O\left(2^{\log ^{1-\varepsilon} n}\right)$ for any fixed $\varepsilon>0$, unless $\mathrm{NP} \subseteq \operatorname{DTIME}\left(n^{\operatorname{polylog}(n)}\right)$.

Recall that CA with $S \neq V$ admits an $O(\ln n)$-approximation algorithm, while for $S=V$ the best known ratio is $O\left(r_{\max } \ln n\right)$ [Kortsarz and Nutov 2008]. Theorem 1.5 shows that for $S=V$ a much better (e.g., a polylogarithmic) approxiamtion algorithm may not exist.

We note that recently Bernáth and Király [Bernáth and Király 2008] showed several extensions and other interesting applications of the new approach developed in this paper, including simplified proofs of many classic results, as well as some new interesting results. It is not clear however that all possible applications of our approach are exhausted.

This paper is organized as follows. In the rest of this section we briefly survey some related work. Theorems 1.3, 1.4, 1.1, and 1.5 are proved in Sections 2, 3, 4, and 5, respectively. In Section 6 we briefly survey some open problems. 


\subsection{Related work}

We briefly summarize the complexity and approximability status of CA problems. For a survey of the cases when polynomial algorithms and good characterizations are available for the minimum see [Frank 2001; 1995]. For directed graphs it was observed in [Frank 1992] that even for rooted $\{0,1\}$-requirements Edge-CA is at least as hard as the Set-Cover problem; rooted requirements means that there is a "root" $s \in V$ so that $r(u, v)>0$ implies $u=s$ or $v=s$. Combined with the result of [Raz and Safra 1997] this implies an $\Omega(\ln n)$-approximation threshold for this simple variant (namely, the problem cannot be approximated within $c \ln n$ for some universal constant $c>0$, unless $\mathrm{P}=\mathrm{NP}$ ). By extending the construction from [Frank 1992], a similar approximation threshold was shown in [Nutov 2006] (see also [Lando and Nutov 2009]) for the undirected Node-CA with rooted $\{0, k\}$-requirements and $k=\Theta(n)$.

We note that CA is a particular case of the Generalized Steiner Network problem, where edges have costs and the goal is to add a min-cost set of edges so that $\lambda_{G+F}^{S}(u, v) \geq r(u, v)$ for all $(u, v) \in V \times V$. For Generalized Steiner Network we may assume that any edge is feasible (if not, assign "infinite" costs to "forbidden" edges). Under this assumption CA is the case of $\{0,1\}$-costs. Another type of costs are the $\{1, \infty\}$-edge costs, so called Min-Size Subgraph Problems. See surveys in [Khuller 1995; Kortsarz and Nutov 2007] on approximation algorithms for various types of Generalized Steiner Network problems.

The Edge-CA: For general $r$, a polynomial algorithm was given by [Frank 1992] based on [Mader 1978] splitting-off theorem. For the min-cost version, [Jain 2001] gave a 2-approximation algorithm.

The Element-CA: Recall that the Hypergraph Edge-CA is a particular case of the Element-CA. [Bang-Jensen and Jackson 1999] showed that the Hypergraph Edge-CA is in $\mathrm{P}$ for uniform requirements $r \equiv k$ on $V \times V$. This result was extended by [Benczúr and Frank 1999] to requirements $r \equiv k$ on $T \times T$ for some $T \subseteq V$ and $r=0$ otherwise. The 2-approximation algorithm of [Jain 2001] for the min-cost version of Edge-CA was extended to the min-cost version of Element-CA by [Fleischer et al. 2006] and by [Cheriyan et al. 2006].

The Node-CA: For $r(u, v) \equiv k$ for all $u, v \in V$ [Jackson and Jordán 2005] gave an algorithm that for any fixed $k$ computes an optimal solution in polynomial time. The complexity status for arbitrary $k$ remains a major open question in graph connectivity (a similar problem for digraphs is solvable in polynomial time [Frank and Jordán 1995]); the best known approximation algorithm due to [Jackson and Jordán 2000] computes a solution with roughly (at most) $k(k-\kappa(G)) / 2$ edges over the optimum, where $\kappa(G)$ is the node connectivity of $G$. As was mentioned, for rooted $\{0, k\}$-requirements in [Nutov 2006] an $\Omega(\ln n)$-approximation threshold was established, and the problem also admits an $O(\ln n)$-approximation algorithm [Kortsarz and Nutov 2008]. However, for requirements $r(s, v)=k$ for all $v \in V-s$ the complexity status of this problem is another open question (for digraphs, this case is in $\mathrm{P}$ even when edges have costs [Frank and Tardos 1989]). For general $r$, no tighter hardness results for the min-size version were known. 


\section{PROOF OF THEOREM 1.3}

In this section we prove Theorem 1.3. Our original proof for the fact that $p$ is $0,1-$ valued in the preliminary version [Nutov 2005] was somewhat long and complicated, and recently [Bernáth and Király 2008] found a much simpler and more constructive proof. We see no point in presenting our original proof, and for this part present a proof along the proof line of [Bernáth and Király 2008].

We say that $X \subset V$ with $p(X)>0$ is dangerous if $g(X) \leq p(X)+1$. Note that if $X$ is dangerous and $u, v \in X \cap T_{g}$, then $g^{u v}(X)=g(X)-2, p^{u v}(X)=p(X)$, and thus $g^{u v}(X)=g(X)-2 \leq p(X)+1-2=p^{u v}(X)-1<p^{u v}(X)$. Hence a pair $u, v$ contained in a dangerous set cannot be legal. It is easy to that that this is the only reason that a pair is not legal, namely, if a pair is not legal then there must be a dangerous set containing it. Thus we have:

Proposition 2.1. A pair $u, v \in T_{g}$ is not $(p, g)$-legal if, and only if, some dangerous set contains $u, v$.

Since $p$ is symmetric, existence of a dangerous set $X$ containing $u, v$ implies $(V-X) \cap T_{g} \neq \emptyset$, hence $\left|T_{g}\right| \geq 3$ must hold. From (1) and (2) it is easy to see that:

Proposition 2.2. Let $p$ be symmetric skew-supermodular, let $\mu=\max \{p(X)$ : $X \subseteq V\}$, and let $\mathcal{F}=\{X \subseteq V: p(X)=\mu\}$. Then $\mathcal{F}$ is symmetric and $X \cap Y, X \cup$ $Y \in \mathcal{F}$ if (1) holds, or $X-Y, Y-X \in \mathcal{F}$ if (2) holds, for any $X, Y \in \mathcal{F}$.

Lemma 2.3. Let $Y \in \mathcal{F}$ (so $p(Y)=\mu$ ) and let $X$ be a dangerous set (so $g(X)$ $1 \leq p(X))$.

(i) If $(X-Y) \cap T_{g} \neq \emptyset$ and if (1) holds then $X \cup Y \in \mathcal{F}$ and $g(X-Y)=1$.

(ii) (ii) If $X \cap Y \cap T_{g} \neq \emptyset$ and if (2) holds then $Y-X \in \mathcal{F}$ and $g(X \cap Y)=1$.

Proof. For (i), note that $(X-Y) \cap T_{g} \neq \emptyset$ implies $g(X \cap Y) \leq g(X)-1$. Thus if (1) holds, then:

$(g(X)-1)+\mu \leq p(X)+p(Y) \leq p(X \cap Y)+p(X \cup Y) \leq g(X \cap Y)+\mu \leq(g(X)-1)+\mu$.

Consequently, equality holds everywhere. Thus $p(X \cup Y)=\mu$ and $g(X \cap Y)=$ $g(X)-1$. This implies $g(X-Y)=1$, as $g(X)=g(X \cap Y)+g(X-Y)$.

For (ii), note that $X \cap Y \cap T_{g} \neq \emptyset$ implies $g(X-Y) \leq g(X)-1$. Thus if (2) holds then:

$(g(X)-1)+\mu \leq p(X)+p(Y) \leq p(X-Y)+p(Y-X) \leq g(X-Y)+\mu \leq(g(X)-1)+\mu$.

Consequently, equality holds everywhere. Thus $p(Y-X)=\mu$ and $g(X-Y)=$ $g(X)-1$. This implies $g(X \cap Y)=1$ as $g(X)=g(X \cap Y)+g(X-Y)$.

Let $Y, Z$ be any inclusion-minimal disjoint sets in $\mathcal{F}$; such exist, by Proposition 2.2. Let $y \in Y \cap T_{g}, z \in Z \cap T_{g}$. We claim that if $\{y, z\}$ is not $(p, g)$-legal then $\mu=1$. Let $X$ be a dangerous set containing $y, z$. By the minimality of $Y$, we must be in Case (i) of Lemma 2.3, so $X \cup Y \in \mathcal{F}$ and $g(X-Y)=1$. By the minimality of $Z$ and Proposition 2.2, we must have $(X \cup Y) \cap Z \in \mathcal{F}$, which is possible only if $Z \subset X$, since $Z, Y$ are disjoint. But then $\mu=p(Z) \leq g(Z) \leq g(X-Y) \leq 1$. Consequently, $p$ is 0,1 -valued. 
Now we prove that $g$ is 0,1 -valued, and that $p_{g}(T)=1$ for all $T \subset T_{g}$ with $|T| \in\{1,2\}$. Recall that $p_{g}(T)=\max \left\{p(X): X \subseteq V, X \cap T_{g}=T\right\}$, hence $p_{g}$ is 0,1 -valued since $p$ is. Let $u, v \in T_{g}$. By Proposition 2.1, there exists a dangerous set $X_{u v}$ containing $u, v$. As $p\left(X_{u v}\right)=1$, we must have $g\left(X_{u v}\right)=2$, so $g(u)=g(v)=1$. This implies that $g$ is 0,1 -valued. Also note that $X_{u v} \cap T_{g}=\{u, v\}$, and that for every $v \in T_{g}$ there exists a set $X_{v}$ with $g\left(X_{v}\right)=p\left(X_{v}\right)=1$, by the minimality of $g$. Consequently, for every $T \subseteq T_{g}$ with $|T| \in\{1,2\}$ there exists $X \subseteq V$ with $X \cap T_{g}=T$ so that $p(X)=1$, hence $p_{g}(T)=1$ for every such $T$.

Finally, we prove the lower bound opt $(p)=\operatorname{opt}\left(p_{g}\right) \geq\left\lceil 2\left|T_{g}\right| / 3\right\rceil$. Let $F$ be a $p_{g}$-cover, so every edge in $F$ has both endnodes in $T_{g}$. To see that $|F| \geq\left\lceil 2\left|T_{g}\right| / 3\right\rceil$, note that in the graph $\left(T_{g}, F\right)$ every connected component must have at least 3 nodes. Indeed, if there is a connected component $T$ in $\left(T_{g}, F\right)$ with $|T| \in\{1,2\}$ then $T$ is not covered by $F$, while $p_{g}(T)>0$, hence $F$ is not a feasible solution.

The proof of Theorem 1.3 is complete.

\section{PROOF OF THEOREM 1.4}

A set $X$ with $p(X)>0$ is $(p, g)$-tight if $p(X)=g(X)$.

Proposition 3.1. Let $p$ be skew-supermodular, let $g$ be a p-transversal, and let $X, Y$ be $(p, g)$-tight sets. Then at least one of the following holds: $X \cap Y, X \cup Y$ are both $(p, g)$-tight (if (1) holds) or $g(X \cap Y)=0$ and $X-Y, Y-X$ are both $(p, g)$-tight (if (2) holds).

Proof. Note that $g(X)+g(Y)=g(X \cap Y)+g(X \cup Y)$ and that $g(X)+g(Y)=$ $g(X-Y)+g(Y-X)+2 g(X \cap Y)$.

If (1) holds then:

$$
\begin{aligned}
g(X)+g(Y) & =p(X)+p(Y) \leq p(X \cap Y)+p(X \cup Y) \leq g(X \cap Y)+g(X \cup Y) \\
& =g(X)+g(Y)
\end{aligned}
$$

Consequently, equality holds everywhere, which implies that $X \cap Y, X \cup Y$ are both $(p, g)$-tight.

If (2) holds then:

$$
\begin{aligned}
g(X)+g(Y) & =p(X)+p(Y) \leq p(X-Y)+p(Y-X) \leq g(X-Y)+g(Y-X) \\
& =g(X)+g(Y)-2 g(X \cap Y) .
\end{aligned}
$$

Consequently, $g(X \cap Y)=0$ and equality holds everywhere, which implies that $X-Y, Y-X$ are both $(p, g)$-tight.

Let $\nu(p)=\max \left\{\sum_{X \in \mathcal{F}} p(X): \mathcal{F}\right.$ is a subpartition of $\left.V\right\}$. Clearly, $g(V) \geq \nu(p)$ and $\operatorname{opt}(p) \geq \nu(p) / 2$ for any $p$ and its transversal $g$, hence the following (known) statement implies opt $(p) \geq g(V) / 2$ for a skew-supermodular $p$.

LEMMA 3.2. If $p$ is skew-supermodular then $g(V)=\nu(p)$ for any minimal $p$ transversal $\mathrm{g}$.

Proof. Among all families of $(p, g)$-tight sets whose union contains $T_{g}$, let $\mathcal{F}$ be one with $\sum_{X \in \mathcal{F}}|X|$ minimal. We claim that the sets in $\mathcal{F}$ are pairwise disjoint. Indeed, by Proposition 3.1, any $X, Y \in \mathcal{F}$ can be replaced either by $X \cup Y$ (if (1) holds), or by $X-Y, Y-X$ (if (2) holds), and the union of the sets in $\mathcal{F}$ will ACM Journal Name, Vol. V, No. N, Month 20YY. 
still contain $T_{g}$. However, if $X, Y \in \mathcal{F}$ intersect, then $|X \cup Y|<|X|+|Y|$ and $|X-Y|+|Y-X|<|X|+|Y|$, so we obtain a contradiction to the minimality of $\sum_{X \in \mathcal{F}}|X|$. Thus $\nu(p) \geq \sum_{X \in \mathcal{F}} p(X)=\sum_{X \in \mathcal{F}} g(X)=g\left(T_{g}\right)=g(V)$. Since $g(V) \geq \nu(p)$, equality holds everywhere, implying $g(V)=\nu(p)$.

If the requirement are exponential in $n$, then a polynomial implementation of algorithms for CA problems is achieved by specifying the multiplicity of every added edge. For an integer $k$, let $k \times u v$ denote a set of $k$ parallel edges between $u$ and $v$. Assuming $k \leq \min \{g(u), g(v)\}$, let $p^{k \times u v}(X)=\max \{p(X)-k, 0\}$ if $|X \cap\{u, v\}|=1$ and $p^{k \times u v}(X)=p(X)$ otherwise; $g^{k \times u v}(x)=g(x)-k$ if $x=u$ or if $x=v$ and $g^{k \times u v}(x)=g(x)$ otherwise. The following algorithm starts with a symmetric skew-supermodular set-function $p$, a minimal $p$-transversal $g$, and a partial solution $F=\emptyset$.

\section{The Legal Pairs Algorithm}

\section{Phase 1:}

While there exists a $(p, g)$-legal pair $u, v$ do:

1. Find the maximum integer $k \leq \min \{g(u), g(v)\}$ so that $g^{k \times u v}$ is a $p^{k \times u v}$-transversal;

EndWhile

2. $g \leftarrow g^{k \times u v}, p \leftarrow p^{k \times u v}, F \leftarrow F+k \times u v ;$

\section{Phase 2:}

$p^{\prime} \leftarrow p, g^{\prime} \leftarrow g ;$

Let $F^{\prime}$ be an inclusion minimal $p^{\prime}$-cover on $T^{\prime}=\left\{v \in V: g^{\prime}(v)=1\right\}$.

Output $F+F^{\prime}$.

If a polynomial oracle for (3) is available, then the algorithm can be implemented in polynomial time. Specifically, $g$ can be computed in polynomial time, and the integer $k$ in the main loop of Phase 1 can be found using binary search; see [Benczúr and Frank 1999] for details.

For the approximation ratio, note that Theorem 1.3 implies $\left|F^{\prime}\right| \leq(3 / 2) \cdot \operatorname{opt}\left(p^{\prime}\right)$. Indeed, opt $\left(p^{\prime}\right) \geq 2\left|T_{g^{\prime}}\right| / 3$ while $\left|F^{\prime}\right| \leq\left|T_{g^{\prime}}\right|$ since $p$ is 0,1 -valued by Theorem 1.3, so $F^{\prime}$ is a forest on $T_{g^{\prime}}$. The ratio $7 / 4$ follows from the following statement by substituting $\beta=3 / 2$.

Claim 3.3. If $\left|F^{\prime}\right| \leq \beta \operatorname{opt}\left(p^{\prime}\right)$ for some $\beta$ then $|F|+\left|F^{\prime}\right| \leq(1+\beta / 2) \operatorname{opt}(p)$.

Proof. By Lemma 3.2, $|F|=g(V) / 2-g^{\prime}(V) / 2=\nu(p) / 2-g^{\prime}(V) / 2$. By Theorem 1.3, $F^{\prime}$ is a forest on $T_{g^{\prime}}$, thus $\left|F^{\prime}\right| \leq g^{\prime}(V)$. Consequently, we have:

$$
\begin{aligned}
|F|+\left|F^{\prime}\right| & =\nu(p) / 2-g^{\prime}(V) / 2+\left|F^{\prime}\right| \leq \nu(p) / 2+\left|F^{\prime}\right| / 2 \leq \operatorname{opt}(p)+\beta \operatorname{opt}\left(p^{\prime}\right) / 2 \\
& \leq(1+\beta / 2) \operatorname{opt}(p)
\end{aligned}
$$

Finally, we prove the APX-hardness of our problem by reducing it to the following problem:

\section{3-Set Packing}

Instance: A set-family $\mathcal{S}$ of subsets of size 3 each of a groundset $V$. 
Objective: Find a subfamily $\mathcal{S}^{\prime} \subseteq \mathcal{S}$ of pairwise disjoint sets (packing) of maximal size.

Given an instance $\mathcal{S}$ of 3-Set Packing let $\mathcal{F}=\{X \subset V: 1 \leq|X| \leq 3, X \notin \mathcal{S}\}$. Let $p(X)=1$ if $X \in \mathcal{F}$ or if $V-X \in \mathcal{F}$, and $p(X)=0$ otherwise. It is easy to see that $p$ is symmetric skew-supermodular, that $g(v)=1$ for all $v \in V$ is the unique minimal $p$-transversal, and that no $(p, g)$-legal pair exists.

Lemma 3.4. $\operatorname{opt}(p)=n-\eta-\lfloor(n-3 \eta) / 4\rfloor$, where $\eta$ is the maximum packing size.

Proof. A partition $\mathcal{T}$ of $V$ is p-separating if $p\left(\cup_{T \in \mathcal{T}}, T\right)=0$ for every $\mathcal{T}^{\prime} \subseteq \mathcal{T}$. It is easy to see that $F$ is a $p$-cover of a 0,1 -valued set-function $p$ on $V$ if, and only if, the partition into connected components of $(V, F)$ is $p$-separating; thus $\operatorname{opt}(p)=|V|-\zeta(p)$, where $\zeta(p)$ is the maximum size of a $p$-separating partition. By the definition of $p$ in the lemma, $\mathcal{T}$ is a $p$-separating partition if, and only if, $|T| \geq 3$ for every $T \in \mathcal{T}$ and if $|T|=3$ then $T \in \mathcal{S}$. Thus a maximum size $p$ separating partition is obtained by adding to a maximum packing $\mathcal{S}^{\prime}$ a maximum collection of sets of size 4 each, except of maybe one of size $>4$. Consequently, $\zeta(p)=\eta+\lfloor(n-3 \eta) / 4\rfloor$, and the statement follows.

By Lemma $3.4 \operatorname{opt}(p)=2 n / 3$ if $\eta=n / 3$. Kann [Khuller 1995] showed that there exists $\varepsilon_{0}>0$ such that the decision problem whether $\eta=n / 3$ or $\eta \leq\left(1-\varepsilon_{0}\right) n / 3$ is NP-complete. In the latter case

$$
\operatorname{opt}(p) \geq n-\frac{\left(1-\varepsilon_{0}\right) n}{3}-\left\lfloor\frac{\varepsilon_{0} n}{4}\right\rfloor \geq \frac{2 n}{3}+\frac{\varepsilon_{0} n}{12}=\frac{2 n}{3}\left(1+\varepsilon_{0} / 8\right) .
$$

Therefore, the decision problem whether opt $(p) \leq 2 n / 3$ or opt $(p) \geq \frac{2 n}{3}\left(1+\varepsilon_{0} / 8\right)$ is also NP-complete. The APX-hardness follows.

The proof of Theorem 1.4 is complete.

\section{PROOF OF THEOREM 1.1}

\subsection{Reducing Element-CA to skew-supermodular Set-Function Edge-Cover}

Edge-CA problems can be casted as edge-cover problems of a supermodular or a skew-supermodular function $p$, c.f., [Frank and Jordán 1995; Benczúr and Frank 1999; Frank 1992; Jain 2001; Fleischer et al. 2006; Cheriyan et al. 2006; Kortsarz and Nutov 2007]. For Edge-CA, an appropriate choice of $p$ is as follows. By Menger's Theorem, $F$ is a feasible solution to Edge-CA if, and only if, $d_{G+F}(X) \geq R(X)$ for all $\emptyset \subset X \subset V$, where $R(X)=\max \{r(u, v): u \in X, v \in V-X\}$ (and $R(\emptyset)=R(V)=0)$. That is

$$
d_{F}(X) \geq p(X)=\max \left\{0, R(X)-d_{G}(X)\right\} \quad \forall \emptyset \subseteq X \subseteq V .
$$

For Element-CA we start with a more general model where $p$ is defined on pairs of subsets of $V$ (see [Frank and Jordán 1995; Fleischer et al. 2006; Cheriyan et al. 2006; Kortsarz and Nutov 2007]). We need the following formulation of Menger's Theorem for $S$-connectivity, that is easily deduced from the original theorem by standard constructions. 
Theorem 4.1 Menger's Theorem for $S$-Connectivity.

Let $u, v$ be two nodes of a (directed or undirected) graph $G=(V, E)$ and let $S \subseteq V$. Then

$$
\begin{aligned}
\lambda_{G}^{S}(u, v)= & \min \{|C|: C \subseteq E+S-\{u, v\}, G-C \text { has no uv-path }\} \\
= & \min \left\{d_{G}\left(X^{\prime}, X^{\prime \prime}\right)+\left|V-\left(X^{\prime}+X^{\prime \prime}\right)\right|: u \in X^{\prime}, v \in X^{\prime \prime},\right. \\
& \left.X^{\prime} \cap X^{\prime \prime}=\emptyset, V-\left(X^{\prime}+X^{\prime \prime}\right) \subseteq S\right\}
\end{aligned}
$$

A setpair is an ordered pair $\left(X^{\prime}, X^{\prime \prime}\right)$ of disjoint subsets of the groundset. An edge set $F$ covers a setpair-function $q$ if $d_{F}\left(X^{\prime}, X^{\prime \prime}\right) \geq q\left(X^{\prime}, X^{\prime \prime}\right)$ for every setpair $\left(X^{\prime}, X^{\prime \prime}\right)$. Element-CA can be casted as a Setpair-Function Edge-Cover problem as follows (c.f., [Frank and Jordán 1995; Fleischer et al. 2006; Cheriyan et al. 2006; Kortsarz and Nutov 2007] for details). Let $(G=(V, E), S, r)$ be an instance of Element-CA. Note that $V-\left(X^{\prime}+X^{\prime \prime}\right) \subseteq S$ if, and only if, $V-S \subseteq X^{\prime}+X^{\prime \prime}$. For a setpair $\left(X^{\prime}, X^{\prime \prime}\right)$ of $V$ with $V-S \subseteq X^{\prime}+X^{\prime \prime}$ let

$$
\begin{gathered}
R\left(X^{\prime}, X^{\prime \prime}\right)=\max \left\{r(u, v): u \in X^{\prime}, v \in X^{\prime \prime}\right\}, \\
\hat{d}_{G}\left(X^{\prime}, X^{\prime \prime}\right)=d_{G}\left(X^{\prime}, X^{\prime \prime}\right)+\left|V-\left(X^{\prime}+X^{\prime \prime}\right)\right| .
\end{gathered}
$$

Given $T \subseteq V$, a setpair $\left(X^{\prime}, X^{\prime \prime}\right)$ is a $T$-setpair if $T \subseteq X^{\prime} \cup X^{\prime \prime}$. By Menger's Theorem for $S$-Connectivity, $F$ is a feasible solution to Element-CA if, and only if, $F$ covers the setpair-function $q$ defined by

$$
q\left(X^{\prime}, X^{\prime \prime}\right)=\max \left\{R\left(X^{\prime}, X^{\prime \prime}\right)-\hat{d}_{G}\left(X^{\prime}, X^{\prime \prime}\right), 0\right\} \quad \forall(V-S) \text {-setpair }\left(X^{\prime}, X^{\prime \prime}\right)
$$

and $q\left(X^{\prime}, X^{\prime \prime}\right)=0$ otherwise.

Definition 4.1. A setpair-function $q$ is symmetric if $q\left(X^{\prime}, X^{\prime \prime}\right)=q\left(X^{\prime \prime}, X^{\prime}\right)$ for every setpair $\left(X^{\prime}, X^{\prime \prime}\right)$, and $q$ is skew-bisupermodular if $q\left(X^{\prime}, X^{\prime \prime}\right)=0$ whenever $X^{\prime}=\emptyset$ and for any setpairs $\left(X^{\prime}, X^{\prime \prime}\right),\left(Y^{\prime}, Y^{\prime \prime}\right)$ with $q\left(X^{\prime}, X^{\prime \prime}\right), q\left(Y^{\prime}, Y^{\prime \prime}\right)>0$ at least one of the following holds:

$$
\begin{gathered}
q\left(X^{\prime}, X^{\prime \prime}\right)+q\left(Y^{\prime}, Y^{\prime \prime}\right) \leq q\left(X^{\prime} \cap Y^{\prime}, X^{\prime \prime} \cup Y^{\prime \prime}\right)+q\left(X^{\prime} \cup Y^{\prime}, X^{\prime \prime} \cap Y^{\prime \prime}\right) \\
q\left(X^{\prime}, X^{\prime \prime}\right)+q\left(Y^{\prime}, Y^{\prime \prime}\right) \leq q\left(X^{\prime} \cap Y^{\prime \prime}, X^{\prime \prime} \cup Y^{\prime}\right)+q\left(X^{\prime \prime} \cap Y^{\prime}, X^{\prime} \cup Y^{\prime \prime}\right) .
\end{gathered}
$$

Let $q$ be a setpair-function on $V$ and let $T \subseteq V$. We say that $q$ is $T$-projectable if $X^{\prime} \cap T, X^{\prime \prime} \cap T$ is a proper partition of $T$ whenever $q\left(X^{\prime}, X^{\prime \prime}\right)>0$.

ClaIm 4.2. Let $q$ be a T-projectable setpair function. If $q$ is skew-bisupermodular then for any $q$-cover $F$ there exists a $q$-cover $F_{T}$ on $T$ so that $\left|F_{T}\right|=|F|$.

Proof. Let $F$ be a $q$-cover and suppose that there is $e=u v \in F$ with $u \notin T$. Let $q^{\prime}$ be the residual setpair function w.r.t. $F^{\prime}=F-e$, namely, $q^{\prime}\left(X^{\prime}, X^{\prime \prime}\right)=$ $\max \left\{q\left(X^{\prime}, X^{\prime \prime}\right)-d_{F^{\prime}}\left(X^{\prime}, X^{\prime \prime}\right), 0\right\}$. It is known that $q^{\prime}$ is symmetric skew-bisupermodular if $q$ is, c.f., [Fleischer et al. 2006; Cheriyan et al. 2006]. Clearly, $e$ covers $q^{\prime}$, hence $q^{\prime}$ is 0,1 -valued. Let $\mathcal{F}=\left\{\left(X^{\prime}, X^{\prime \prime}\right): q^{\prime}\left(X^{\prime}, X^{\prime \prime}\right)=1\right\}$. As $e$ covers $q^{\prime}$, we have $u \in X^{\prime}$ and $v \in X^{\prime \prime}$, or $v \in X^{\prime}$ and $u \in X^{\prime \prime}$, for any $\left(X^{\prime}, X^{\prime \prime}\right) \in \mathcal{F}$. Let $\left(X^{\prime}, X^{\prime \prime}\right) \in \mathcal{F}$ be with $u \in X^{\prime}$ and $X^{\prime} \cap T$ inclusion minimal, and let $t \in X^{\prime} \cap T$; such $t$ exists, since $q$, and thus also $q^{\prime}$ is $T$-projectable, hence $X^{\prime} \cap T, X^{\prime \prime} \cap T$ is a proper partition of $T$ for any $\left(X^{\prime}, X^{\prime \prime}\right) \in \mathcal{F}$. We claim that $t v$ covers $q^{\prime}$. Let 
$\left(Y^{\prime}, Y^{\prime \prime}\right) \in \mathcal{F}$. Since $q^{\prime}$ is skew-bisupermodular, and since $u v$ covers $q^{\prime}$ we have: $\left(X^{\prime} \cap Y^{\prime}, X^{\prime \prime} \cup Y^{\prime \prime}\right),\left(X^{\prime} \cup Y^{\prime}, X^{\prime \prime} \cap Y^{\prime \prime}\right) \in \mathcal{F}$ if $u \in Y^{\prime}$ and $v \in Y^{\prime \prime}$, or $\left(X^{\prime} \cap Y^{\prime \prime}, X^{\prime \prime} \cup Y^{\prime}\right),\left(X^{\prime} \cup Y^{\prime \prime}, X^{\prime \prime} \cap Y^{\prime}\right) \in \mathcal{F}$ if $v \in Y^{\prime}$ and $u \in Y^{\prime \prime}$.

By the minimality of $X^{\prime} \cap T$, we must have $X^{\prime} \cap T \subseteq X^{\prime} \cap Y^{\prime} \cap T$ in the former case and $X^{\prime} \cap T \subseteq X^{\prime} \cap Y^{\prime \prime} \cap T$ in the latter case. In each one of the cases, $t v$ covers $\left(Y^{\prime}, Y^{\prime \prime}\right)$. Thus $t v$ covers $q^{\prime}$, and this implies that $F^{\prime}=F-e+t v$ is a $q$-cover. We repeat this replacement procedure for any edge that has an endnode in $V-T$, until obtaining a $q$-cover $F_{T}$ on $T$ with $\left|F_{T}\right|=|F|$.

If $q$ is $T$-projectable, then the projection of $q$ on $T$ is a set-function $p$ on $T$ defined by

$$
p(X)=\max \left\{q\left(X^{\prime}, X^{\prime \prime}\right): X^{\prime} \cap T=X\right\}
$$

ClaIm 4.3. Let $q$ be a T-projectable setpair-function, and let $p$ be the projection of $q$ on $T$. Then $p$ is symmetric if $q$ is, and $p$ is skew-supermodular if $q$ is skewbisupermodular.

Proof. From the definition of $p$ it follows that $p$ is symmetric if $q$ is. We prove that $p$ is skew-supermodular if $q$ is skew-bisupermodular. Let $\left(X^{\prime}, X^{\prime \prime}\right),\left(Y^{\prime}, Y^{\prime \prime}\right)$ be setpairs so that $q\left(X^{\prime}, X^{\prime \prime}\right)=p(X)>0$ and $q\left(Y^{\prime}, Y^{\prime \prime}\right)=p(Y)>0$, where $X=X^{\prime} \cap T$ and $Y=Y^{\prime} \cap T$.

If (5) holds then:

$$
\begin{aligned}
p(X)+p(Y) & =q\left(X^{\prime}, X^{\prime \prime}\right)+q\left(Y^{\prime}, Y^{\prime \prime}\right) \leq \\
& \leq q\left(X^{\prime} \cap Y^{\prime}, X^{\prime \prime} \cup Y^{\prime \prime}\right)+q\left(X^{\prime} \cup Y^{\prime}, X^{\prime \prime} \cap Y^{\prime \prime}\right) \leq \\
& \leq p\left(\left(X^{\prime} \cap Y^{\prime}\right) \cap T\right)+p\left(\left(X^{\prime} \cup Y^{\prime}\right) \cap T\right)=p(X \cap Y)+p(X \cup Y) .
\end{aligned}
$$

If (6) holds then:

$$
\begin{aligned}
p(X)+p(Y) & =q\left(X^{\prime}, X^{\prime \prime}\right)+q\left(Y^{\prime}, Y^{\prime \prime}\right) \leq \\
& \leq q\left(X^{\prime} \cap Y^{\prime \prime}, X^{\prime \prime} \cup Y^{\prime}\right)+q\left(X^{\prime \prime} \cap Y^{\prime}, X^{\prime} \cup Y^{\prime \prime}\right) \leq \\
& \leq p\left(\left(X^{\prime} \cap Y^{\prime \prime}\right) \cap T\right)+p\left(\left(X^{\prime \prime} \cap Y^{\prime}\right) \cap T\right)=p(X-Y)+p(Y-X) .
\end{aligned}
$$

This implies that $p$ is skew-supermodular if $q$ is skew-bisupermodular.

Claims 4.2 and 4.3 imply an approximation ratio preserving reduction from Setpair-Function Edge-Cover with skew-bisupermodular $q$ to Set-Function Edge-Cover with the skew-supermodular projection $p$ on $T$ (provided $q$ is $T$-projectable).

The 7/4-approximation in Theorem 1.1 follows. Let $T=V-S$. For Element-CA, the setpair-function $q$ in (4) is symmetric skew-bisupermodular, and $T$-projectable. Claims 4.2 and 4.3 imply an approximation ratio preserving reduction to SetFunction Edge-Cover with skew-supermodular $p$, where $p$ is the projection of $q$ on $T=V-S$. It is not hard to verify that via max-flows a minimal $p$-transversal $g$ can be found in polynomial time, and that (3) for $p$ can be realized in polynomial time. Consequently, Theorem 1.4 implies that the Legal Pairs Algorithm applied on $p$ is a $7 / 4$-approximation algorithm for Element-CA.

Note that symmetric skew-supermodular set-functions are a particular case of symmetric skew-bisupermodular setpair functions. Namely, if $p$ is a set function then the corresponding setpair-function is $q(X, V-X)=p(X)$ and $q\left(X^{\prime}, X^{\prime \prime}\right)=0$ 
otherwise. Note also that with this correspondence any symmetric set-function $p$ is $T_{g}$-projectable, for any $p$-transversal $g$. Hence Claims 4.2 and 4.3 with $T=T_{g}$ imply Lemma 1.2 from the Introduction.

Remark: For Element-CA with $r(u, v)=k$ on $T \times T$ for some $T \subseteq V$ the set function $q$ in (4) is symmetric bisupermodular, namely, (5) holds for any setpairs $\left(X^{\prime}, X^{\prime \prime}\right),\left(Y^{\prime}, Y^{\prime \prime}\right)$ with $q\left(X^{\prime}, X^{\prime \prime}\right), q\left(Y^{\prime}, Y^{\prime \prime}\right)>0$. The projection $p$ of $q$ on $T$ is symmetric supermodular, by the first case in the proof of Claim 4.3. Thus Claim 4.2 implies that the algorithm of Benczúr and Frank [Benczúr and Frank 1999] applied on $p$ solves this version of Element-CA.

Via the following well known reduction our algorithms apply also for Hypergraph Edge-CA. Recall that in the Hypergraph Edge-CA the goal is to augment a hypergraph $H=(U, S)$ by edges of size two to meet prescribed pairwise (hyper)edge connectivity requirements. For basic definitions on hypergraphs we refer the reader to [Duchet 1995].

Proposition 4.4. The Hypergraph Edge-CA admits a polynomial time approximation ratio preserving reduction to the Element-CA.

Proof. A bipartite graph $G=(U+S, E)$ is the incidence graph of a hypergraph $H=(U, S)$ if $E=\{u s: u \in s \in S\}$. Thus, for every instance $\mathcal{I}_{H}=(H=(U, S), r)$ of hypergraph edge-CA corresponds an instance $\mathcal{I}_{G}=(G=(U+S, E), r)$ of elementCA. Furthermore, if $\lambda_{H}(u, v)$ is the maximum number of hyperedge disjoint $u v$ paths in $H$, then for any edge set $F$ on $U$ we have $\lambda_{H+F}(u, v)=\lambda_{G+F}^{S}(u, v)$ for all $u, v \in U$. That is, an edge set $F_{U}$ on $U$ is a feasible solution to $\mathcal{I}_{H}$ if, and only if, $F_{U}$ is a feasible solution to $\mathcal{I}_{G}$. This reduces the hypergraph Edge-CA to an instance of Element-CA with a restriction that adding edges incident to nodes in $S$ is not allowed. Claim 4.3 shows that by dropping this restriction we get an equivalent problem. That is, the Hypergraph Edge-CA is equivalent to the restriction of Element-CA to bipartite graphs with parts $S, V-S$.

Remark: The min-cost Hypergraph Edge-CA admits a similar polynomial time approximation ratio preserving reduction to the min-cost Element-CA. For that, take $G$ to be the incidence graph of $H$, and set to infinity the costs of the edges incident $S$. For example, as the min-cost Element-CA admits a 2-approximation algorithm [Fleischer et al. 2006; Cheriyan et al. 2006] we get the same result for the min-cost Hypergraph Edge-CA. The inverse reduction does not seem to work, since in the Element-CA we might be allowed to add edges incident to nodes in $S$, while no such edges can exist in Hypergraph Edge-CA.

\subsection{A 3/2-approximation for Element-CA with $r(u, v) \in\{0,1,2\}$}

Let $(G=(V, E), S, r)$ be an instance of Element-CA, and let $D=\{u v: r(u, v)-$ $\left.\lambda_{G}^{S}(u, v) \geq 1\right\}$ be the "demands" that are not satisfied. Clearly, we may and will assume that $r(u, v)=0$ if $u v \notin D$. Let $q$ be the setpair-function defined by (4), let $p$ be the projection of $q$ on $T=V-S$, let $g$ be a minimal $p$-transversal, and let $p_{g}$ be the projection of $p$ on $T_{g}$.

For a (connected) component $H$ of $G$ let $\alpha(H)=\max \{r(u, v): u, v \in V,\{u, v\} \cap$ $H \neq \emptyset\}$. Following [Frank 1992], we say that $H$ is a marginal component if $r(u, v) \leq$ 
$\lambda_{G}^{S}(u, v)$ for any $u, v \in H$ and $R(H, V-H) \leq 1$. If $r(u, v) \in\{0,1,2\}$ and $r(u, v)=0$ whenever $u v \notin D$, this is equivalent to $\alpha(H) \leq 1$. It is easy to see that if $\alpha(H)=0$ then $\left|H \cap T_{g}\right|=0$ and $H$ can be eliminated.

The algorithm is based on the following statement, which is of independent interest:

THEOREM 4.5. Suppose that no $(p, g)$-legal pair exists for an instance of ElementCA with $r(u, v) \in\{0,1,2\}$. Then $G$ has at most one non-marginal component. If $G$ has a non-marginal component $H$ with $\left|H \cap T_{g}\right| \geq 4$ then there is $s \in S \cap H$ so that $\left|C \cap T_{g}\right| \leq 1$ for any component $C$ of $H-s$.

It is not hard to verify that Theorem 4.5 implies:

COROLlary 4.6. Under the assumptions of Theorem 4.5, let $\mathcal{T}$ be the partition of $T_{g}$ induced by the components of $G+D-s$, where $s$ is as in Theorem 4.5 if $\left|H \cap T_{g}\right| \geq 4$ and $s=\emptyset$ if $\left|H \cap T_{g}\right| \leq 3$ or if all components of $G$ are marginal. Then $p_{g}(X)=1$ for $X \subset T_{g}$ if, and only if, $X$ divides some part of $\mathcal{T}$. Consequently, $\operatorname{opt}\left(p_{g}\right)=\left|T_{g}\right|-|\mathcal{T}|$, and any inclusion minimal or optimal $p_{g}$-cover is a union of trees on the parts of $\mathcal{T}$.

A 3/2-approximation algorithm for Element-CA with $r(u, v) \in\{0,1,2\}$ follows by substituting $\beta=1$ in Claim 3.3, as in Phase 2 of the Legal Pairs Algorithm an optimal solution is found.

In the rest of this section we prove Theorem 4.5. Assume that no $(p, g)$-legal pair exists. By Theorem 1.3 and the definition of $p$ we have that $u v \in D$ implies $r(u, v)-\lambda_{G}^{S}(u, v)=1$, and:

Claim 4.7. $q\left(X^{\prime}, X^{\prime \prime}\right)=1$ for a setpair $\left(X^{\prime}, X^{\prime \prime}\right)$ if, and only if, exactly one of the following holds:

(i) $R\left(X^{\prime}, X^{\prime \prime}\right)=1, \delta\left(X^{\prime}, X^{\prime \prime}\right)=\emptyset$, and $X^{\prime}, X^{\prime \prime}$ is a partition of $V$.

(ii) $R\left(X^{\prime}, X^{\prime \prime}\right)=2, \delta\left(X^{\prime}, X^{\prime \prime}\right)=\{e\}$ for some $e \in E$ and $X^{\prime}, X^{\prime \prime}$ partition $V$.

(iii) $R\left(X^{\prime}, X^{\prime \prime}\right)=2, \delta\left(X^{\prime}, X^{\prime \prime}\right)=\emptyset$ and $V-\left(X^{\prime} \cup X^{\prime \prime}\right)=\{s\}$ for some $s \in S$.

Claim 4.8. Let $P_{i}$ be a $u_{i} v_{i}$-path in $G, i=1,2$, for distinct $u_{1}, v_{1}, u_{2}, v_{2} \in T_{g}$. Then $P_{1}, P_{2}$ share a node $s \in S$ and have no other node in common.

Proof. By Theorem 1.3 there is $X \subset V-S$ with $X \cap T_{g}=\left\{u_{1}, u_{2}\right\}$ and $p(X)=1$. Let $\left(X^{\prime}, X^{\prime \prime}\right)$ be a setpair with $q\left(X^{\prime}, X^{\prime \prime}\right)=1$ and $X^{\prime} \cap T=X$. In particular, $X^{\prime} \cap T_{g}=\left\{u_{1}, u_{2}\right\}$ and $v_{1}, v_{2} \in X^{\prime \prime}$. As $P_{1}, P_{2}$ are paths between $X^{\prime}$ and $X^{\prime \prime}$, (i) in Claim 4.7 cannot hold. Thus we have:

(*) $P_{1}, P_{2}$ share: an edge (if (ii) in Claim 4.7 holds), or a node $s \in S$ (if (iii) in Claim 4.7 holds).

It is not hard to verify that if $P_{1}, P_{2}$ have an edge or a node not from $S$ in common, then there are a $u_{1} u_{2}$-path and a $v_{1} v_{2}$-path, or there are a $u_{1} v_{2}$-path and a $u_{2} v_{1}$ path, that do not have an edge or a node in $S$ in common. In both cases we obtain a contradiction to $\left(^{*}\right)$ for some other pair.

Claim 4.9. $\left|H \cap T_{g}\right| \geq 2$ if $\alpha(H)=2$, and $\left|H \cap T_{g}\right|=\alpha(H)$ if $\alpha(H) \in\{0,1\}$.

Proof. Suppose that $\alpha(H)=2$. Then $r(u, v)=2$ for some $u, v \in H$ with $u v \in D$. We prove that $\left|H \cap T_{g}\right| \geq 2$. Let $\left(X^{\prime}, X^{\prime \prime}\right)$ be a setpair with $u \in X^{\prime}, v \in X^{\prime \prime}$, ACM Journal Name, Vol. V, No. N, Month 20YY. 
and $\hat{d}\left(X^{\prime}, X^{\prime \prime}\right)=\lambda_{G}^{S}(u, v)=1$. It is easy to see that then $\hat{d}\left(X^{\prime} \cap H, X^{\prime \prime} \cup\left(X^{\prime}-H\right)\right)=$ $\hat{d}\left(X^{\prime \prime} \cap H, X^{\prime} \cup\left(X^{\prime \prime}-H\right)\right)=1$. Thus each of $X^{\prime} \cap H, X^{\prime \prime} \cap H$ contains a node from $T_{g}$.

Suppose that $\alpha(H) \in\{0,1\}$. As we assume that no $(p, g)$-legal pair exists, $p$ and $g$ are 0,1 -valued, by Theorem 1.3. Thus by the minimality of $g$, for every $v \in T_{g}$ there exists a set $X_{v}$ with $g\left(X_{v}\right)=p\left(X_{v}\right)=1$. Consequently, $T_{g}$ is obtained by picking one node from every minimal member of $\mathcal{F}=\{X \subset V-S: p(X)=1\}$. It is easy to see that if $\alpha=1$ then $H-S$ is a minimal member of $\mathcal{F}$, and if $\alpha(H)=0$ then any minimal member of $\mathcal{F}$ is disjoint to $H$.

Claim 4.10. G has at most one non-marginal component.

Proof. Suppose to the contrary that $G$ has non-marginal components $H_{1} \neq H_{2}$. Then by Claim 4.9, $\left|H_{1} \cap T_{g}\right|,\left|H_{2} \cap T_{g}\right| \geq 2$. Let $u_{1}, v_{1} \in H_{1} \cap T_{g}$ and $u_{2}, v_{2} \in H_{2} \cap T_{g}$. Let $P_{i}$ be a $u_{i} v_{i}$-path, $i=1,2$. Then $P_{1}, P_{2}$ have no edge or node in common, contradicting Claim 4.8.

Claim 4.11. Suppose that $G$ has a non-marginal component $H$. If $\left|H \cap T_{g}\right| \geq 4$ then there is $s \in S \cap H$ so that $\left|C \cap T_{g}\right| \leq 1$ for any component $C$ of $H-s$.

Proof. It is sufficient to show that if $\left|H \cap T_{g}\right| \geq 4$ then there is $s \in S \cap H$ so that $s$ belongs to any $u v$-path $P$ with $u, v \in T_{g}$. Let $P_{1}, P_{2}$ and $s$ be as in Claim 4.8, let $\left\{u^{\prime}, v^{\prime}\right\}=\left\{u_{1}, v_{1}, u_{2}, v_{2}\right\}-\{u, v\}$, and let $P^{\prime}$ be a $u^{\prime} v^{\prime}$-path in $H$. By Claim 4.8, $P$ and $P^{\prime}$ share a node $s^{\prime} \in S$ and have no other node in common. One can easily verify that if $s^{\prime} \neq s$ then there is a pair of paths between disjoint pairs in $T_{g}$ that have an edge in common, contradicting Claim 4.8.

Remark. Claim 4.11 is not true if $\left|H \cap T_{g}\right|=3$ even for edge-connectivity (the case $S=\emptyset)$. For example:

$$
\begin{aligned}
& V=\left\{v_{1}, v_{2}, v_{3}, t_{1}, t_{2}, t_{3}\right\}, \\
& E=\left\{v_{1} v_{2}, v_{2} v_{3}, v_{3} v_{1}, v_{1} t_{1}, v_{2} t_{2}, v_{3} t_{3}\right\},
\end{aligned}
$$

$r\left(t_{i}, t_{j}\right)=2$, and $T_{g}=\left\{t_{1}, t_{2}, t_{3}\right\}$.

The proof of Theorem 4.5 is complete.

Remark. In the preliminary version [Nutov 2005] the author also claimed that the Legal Pairs Algorithm has approximation ratio $3 / 2$ for Element-CA with $r(u, v) \in$ $\{0, k\}$. This result is correct, but its full proof is long and complicated, and will be presented elsewhere. Specifically, the proof is based on the following analogue of Theorem 4.5 and Corollary 4.6:

THEOREM 4.12. Suppose that no $(p, g)$-legal pair exists for an instance of Element-CA with $r(u, v) \in\{0, k\}$. Then $\lambda_{G}^{S}(u, v)=k-1$ for all $u, v \in T_{g}$, and there exists $K \subseteq S$ with $|K|=k-1$ so that $\left|C \cap T_{g}\right| \leq 1$ for any component $C$ of $G-K$. Furthermore, let $\mathcal{T}$ be the partition of $T_{g}$ induced by the components of $G+D-K$. Then $|T| \geq 3$ for all $T \in \mathcal{T}$, and $p_{g}(X)=1$ for $X \subset T_{g}$ if, and only if, $X$ divides some part of $\mathcal{T}$. Consequently, $\operatorname{opt}\left(p_{g}\right)=\left|T_{g}\right|-|\mathcal{T}|$, and any inclusion minimal or optimal $p_{g}$-cover is a union of trees on the parts of $\mathcal{T}$. 


\section{PROOF OF THEOREM 1.5}

To prove Theorem 1.5, we reduce the Node-CA with $r(u, v) \in\{0, k\}$ to the following problem:

Min-Rep:

Instance: A bipartite graph $H=(A+B, I)$, and equitable partitions $\mathcal{A}$ of $A$ and $\mathcal{B}$ of $B$.

Objective: Find a minimum size node set $A^{\prime} \cup B^{\prime}$, where $A^{\prime} \subseteq A$ and $B^{\prime} \subseteq B$, so that for any $A_{i} \in \mathcal{A}, B_{j} \in \mathcal{B}$ with $\delta_{I}\left(A_{i}, B_{j}\right) \neq \emptyset$ there are $a \in A^{\prime} \cap A_{i}$ and $b \in B^{\prime} \cap B_{j}$ so that $a b \in I$.

Let $H$ be a graph on a node set $V$. Recall that $\kappa_{H}(u, v)=\lambda_{H}^{V}(u, v)$ for $u, v \in V$. For $X \subseteq V$ let $\Gamma_{H}(X)$ denote the set of neighbors of $X$ in $H$. An instance of Min-Rep has the star property if $\left|\Gamma_{H}(b) \cap A_{i}\right| \leq 1$ holds for every $b \in B$ and any $A_{i} \in \mathcal{A}$. The following statement follows directly from the Parallel Repetition Theorem of [Raz 1998], that considered hardness of approximation for the so called Label-Cover-Max problem, e.g., see [Kortsarz et al. 2004, Theorem 2.1].

THEOREM 5.1. Min-Rep with star property on instances with $n$ nodes cannot be approximated within $O\left(2^{\log ^{1-\varepsilon} n}\right)$ for any $\varepsilon>0$, unless $\mathrm{NP} \subseteq \operatorname{DTIME}\left(n^{\operatorname{polylog}(n)}\right)$.

The proof of Theorem 1.5 follows. Given an instance $(H=(A+B, I), \mathcal{A}, \mathcal{B})$ of Min-Rep we construct an instance $(G=(V, E), r)$ of node-CA as follows. Let

$$
\mathcal{E}=\left\{i j: A_{i} \in \mathcal{A}, B_{j} \in \mathcal{B}, \delta_{H}\left(A_{i}, B_{j}\right) \neq \emptyset\right\}
$$

The graph $G=(V, E)$ is obtained from $H$ as follows.

(1) Add to $H$ : a set $\left\{a_{1}, \ldots, a_{|\mathcal{A}|}, b_{1}, \ldots, b_{|\mathcal{B}|}\right\}$ of $|\mathcal{A}|+|\mathcal{B}|$ nodes, and for every $i j \in \mathcal{E}$ a pair of nodes $a_{i j}, b_{i j}$ (so a total number of nodes added to $H$ is $|\mathcal{A}|+|\mathcal{B}|+2|\mathcal{E}|)$. Thus

$$
V=A+B+\left\{a_{1}, \ldots, a_{|\mathcal{A}|}, b_{1}, \ldots, b_{|\mathcal{B}|}\right\}+\left\{a_{i j}: i j \in \mathcal{E}\right\}+\left\{b_{i j}: i j \in \mathcal{E}\right\} .
$$

and $|V|=(|A|+|B|)+(|\mathcal{A}|+|\mathcal{B}|+2|\mathcal{E}|)$.

(2) For every $i j \in \mathcal{E}$ connect: $a_{i j}$ to every node that is not in $\bar{A}_{i j}=A_{i}+B_{j}+b_{j}+b_{i j}$, and $b_{i j}$ to every node that is not in $\bar{B}_{i j}=A_{i}+B_{j}+a_{i}+a_{i j}$. Thus

$$
E=I+\left\{a_{i j} w: i j \in \mathcal{E}, w \in V-\bar{A}_{i j}\right\}+\left\{b_{i j} w: i j \in \mathcal{E}, w \in V-\bar{B}_{i j}\right\} .
$$

For $i j \in \mathcal{E}$ let

$$
C_{i j}=V-\left(\bar{A}_{i j}+\bar{B}_{i j}\right)=\Gamma_{G}\left(a_{i j}\right) \cap \Gamma_{G}\left(b_{i j}\right) .
$$

Since the partitions $\mathcal{A}, \mathcal{B}$ are equitable, the sets $C_{i j}$ are all of the same size, say $k-1$. Every node in $C_{i j}$ is an internal node of an $a_{i j} b_{i j}$-path of length 2 . By the construction, in $G_{i j}=G-C_{i j}$ there is no $a_{i j} b_{i j}$-path. Thus $C_{i j}$ is a minimum node cut separating $a_{i j}$ and $b_{i j}$ and

$$
\kappa_{G}\left(a_{i j}, b_{i j}\right)=k-1 \text { for } i j \in \mathcal{E} .
$$

The requirement function is defined by:

$$
r\left(a_{i j}, b_{i j}\right)=k \text { for } i j \in \mathcal{E}
$$

ACM Journal Name, Vol. V, No. N, Month 20YY. 
and $r(u, v)=0$ otherwise. Clearly, the construction is polynomial.

For an edge set $F$ and $i j \in \mathcal{E}$ let $F_{i j}$ be the edges in $F$ with both endnodes in $G_{i j}$. Clearly:

Claim 5.2. For every $i j \in \mathcal{E}$ there is no $a_{i j} b_{i j}$-path in $G_{i j}$, and a set $F$ of edges is a feasible solution to $(G, r)$ if, and only if, $G_{i j}+F_{i j}$ contains an $a_{i j} b_{i j}$-path for every ij $\in \mathcal{E}$.

An edge is proper if it connects $b_{j}$ to some node in $B_{j}$, or $a_{i}$ to some node in $A_{i}$.

Claim 5.3. Let $F$ be a feasible solution to $(G, r)$. If $e \in F$ is a non-proper edge then there exist proper edges $e^{\prime}, e^{\prime \prime}$ such that $F-e+\left\{e^{\prime}, e^{\prime \prime}\right\}$ is also a feasible solution to $(G, r)$. Thus there exists a proper feasible solution $F^{\prime}$ with $\left|F^{\prime}\right| \leq 2|F|$.

Proof. Assume that $F-e$ is not a feasible solution, as otherwise the statement is trivial. Then, by Claim 5.2, one of the following three cases holds, where for each case we indicate an appropriate choice of $e^{\prime}, e^{\prime \prime}$ :

- $e \in \delta_{F}\left(A_{i}+a_{i}+a_{i j}, B_{j}+b_{j}+b_{i j}\right)$ for some $i j \in \mathcal{E}$ :

in this case set $\left\{e^{\prime}, e^{\prime \prime}\right\}=\left\{a_{i} a, b_{j} b\right\}$, for some $a b \in I$ (such edge $a b$ exists, since $i j \in \mathcal{E})$.

- $e=a_{i j} a$ for some $a \in A_{i}$ or $e=b_{i j} b$ for some $b \in B_{j}$ : set $e^{\prime}=e^{\prime \prime}=a_{i} a$ or $e^{\prime}=e^{\prime \prime}=b_{j} b$, respectively.

- $e=a^{\prime} a^{\prime \prime}$ for some $a^{\prime}, a^{\prime \prime} \in A_{i}$ or $e=b^{\prime} b^{\prime \prime}$ for some $b^{\prime}, b^{\prime \prime} \in B_{j}$ : set $\left\{e^{\prime}, e^{\prime \prime}\right\}=\left\{a_{i} a^{\prime}, a_{i} a^{\prime \prime}\right\}$ or $\left\{e^{\prime}, e^{\prime \prime}\right\}=\left\{b_{j} b^{\prime}, b_{j} b^{\prime \prime}\right\}$, respectively.

In each one of the cases, it is easy to see that for any $i j \in \mathcal{E}$ with $e \in F_{i j}$ holds: the endnodes of $e^{\prime}, e^{\prime \prime}$ are nodes of $G_{i j}$, and in $G_{i j}+\left(F_{i j}-e+\left\{e^{\prime}, e^{\prime \prime}\right\}\right)$ there is an $a_{i j} b_{i j}$-path. Thus $F-e+\left\{e^{\prime}, e^{\prime \prime}\right\}$ is a feasible solution as well, by Claim 5.2.

Claim 5.4. A proper edge set $F$ is a feasible solution to $(G, r)$ if, and only if, the end-nodes of $F$ contained in $A+B$ is a feasible solution to the original Min-Rep instance.

Proof. Note that for every node $v \in A+B$ naturally corresponds a unique proper edge, namely, $a_{i} v$ if $v \in A_{i}$ and $b_{j} v$ if $v \in B_{j}$. Thus there is a bijective correspondence between proper edge sets and subsets $A^{\prime}+B^{\prime}$ of $A+B$, where $A^{\prime} \subseteq A, B^{\prime} \subseteq B$. Let $A^{\prime}+B^{\prime}$ and $F$ be such corresponding pair. Recall that $A^{\prime}+B^{\prime}$ is a feasible solution to Min-Rep if, and only if, for every $i j \in \mathcal{E}$ there are $a \in A^{\prime} \cap A_{i}, b \in B^{\prime} \cap B_{j}$ such that $a b \in I$. Note that for $i j \in \mathcal{E}$ there are such $a, b$ if, and only if, there is an $a_{i j} b_{i j}$-path $a_{i j}, a_{i}, a, b, b_{j}, b_{i j}$ of length 5 in $G_{i j}+F_{i j}$; this is true since our Min-Rep instance has the star property. The statement now follows from Claim 5.2.

Since in the construction $|V|=O\left(n^{2}\right)$, where $n=|A|+|B|$, Theorem 5.1 implies Theorem 1.5.

Remark: The best known approximation ratio for Min-Rep is $O(\sqrt{|A|+|B|})$ Peleg 2007]. Claim 5.4 implies that if Min-Rep with star property has "approximation threshold" $(|A|+|B|)^{\varepsilon}$, then the node-CA has "approximation threshold" $(|A|+|B|)^{\varepsilon}=\Omega\left(|V|^{\varepsilon / 2}\right)$. 


\section{OPEN PROBLEMS}

One open problem is to improve the hardness results for Element-CA (the current one just states that the problem is NP-hard) or the approximation ratio. Another open problem is whether we can compute in polynomial time an optimal solution for general instances of Element-CA without legal pairs; if so, then this will give a 3/2approximation algorithm. Note that Theorem 1.4 does not imply APX-hardness for Set-Function Edge-Cover instances arising from Element-CA instances.

For Set-Function Edge-Cover with skew supermodular $p$, we conjecture that better ratios can be achieved than the ones given in this paper; our goal was just to break the 2 barrier. Specifically, at Phase 1 of the Legal Pairs Algorithm a more advanced strategy is to split-off pairs while trying to minimize the edge-set $F^{\prime}$ computed at Phase 2. It is also possible that our ratio of $3 / 2$ on instances without legal pairs can be improved.

For Node-CA we have shown an approximation threshold $\Omega\left(2^{\left.\log ^{1-\epsilon} n\right)}\right)$ for $\{0, k\}$ requirements, while in [Kortsarz and Nutov 2008] an $r_{\max } \cdot O(\log n)$-approximation algorithm was shown for arbitrary requirements. Note that $k=\Theta(n)$ in the reduction we used, while for undirected graphs and small values of $k$ constant approximation guarantees can be achieved. In the preliminary version [Nutov 2005] the author posed the following two questions:

(1) Can one achieve a constant approximation ratio for (undirected) Node-CA with $k=r_{\max }$ bounded by a constant, namely, does the problem admits a $\rho(k)$ approximation algorithm, where $\rho(k)$ is a function of $k$ only?

(2) Can one a achieve an approximation ratio $O\left(n^{1-\varepsilon}\right)$ for Node-CA?

Recently, the author answered the first question in [Nutov 2008], obtaining for Node-CA approximation ratios $O\left(k \ln ^{2} k\right)$ for arbitrary requirements and $O\left(\ln ^{2} k\right)$ for rooted requirements. The second question still remains open.

\section{Acknowledgment:}

I thank two anonymous referees for many useful comments.

\section{REFERENCES}

BAng-Jensen, J. AND JACKSON, B. 1999. Augmenting hypergraphs with edges of size two. Math. Programming 84, 467-481.

Benczúr, A. And Frank, A. 1999. Covering symmetric supermodular functions by graphs. Math. Programming 84, 483-503.

Bernáth, A. And KirÁLy, T. 2008. A new approach to splitting-off. EGRES Technical Report No. 2008-02.

Cheriyan, J., Vempala, S., And Vetta, A. 2006. Network design via iterative rounding of setpair relaxations. Combinatorica 26, 3, 255-275.

Cosh, B., Jackson, B., And Kiraly, Z. 2003. Local connectivity augmentation in hypergraphs is NP-complete. Manuscript.

Duchet, P. 1995. Hypergraphs. In Chapter 7 in Handbook of Combinatorics, R. L. Graham, M. Grötschel, and L. Lovász, Eds. MIT Press, Cambridge, MA, USA.

Fleischer, L., Jain, K., AND Williamson, D. P. 2006. Iterative rounding 2-approximation algorithms for minimum-cost vertex connectivity problems. J. of Computer and System Sciences 72, 5, 838-867.

FrANK, A. 1992. Augmenting graphs to meet edge-connectivity requirements. SIAM J. on Discrete Mathematics 5, 1, 25-53.

ACM Journal Name, Vol. V, No. N, Month 20YY. 
Frank, A. 1995. Connectivity and network flows. In Chapter 2 in Handbook of Combinatorics, R. L. Graham, M. Gr"otschel, and L. Lovász, Eds. MIT Press, Cambridge, MA, USA.

Frank, A. 2001. Edge-connection of graphs, digraphs, and hypergraphs. EGRES TR No 2001-11.

Frank, A. AND Jordán, T. 1995. Minimal edge-coverings of pairs of sets. J. on Combinatorial Theory B 65, 73-110.

Frank, A. And Tardos, E. 1989. An application of submodular flows. Linear Algebra and its Applications 114/115, 329-348.

JACKSON, B. AND JordÁN, T. 2000. A near optimal algorithm for vertex connectivity augmentation. In ISAAC. Springer-Verlag, London, UK, 312-325.

JACKSON, B. AND JoRDÁN, T. 2005. Independence free graphs and vertex connectivity augmentation. J. on Combinatorial Theory B 94, 1, 31-77.

JAIN, K. 2001. A factor 2 approximation algorithm for the generalized Steiner network problem. Combinatorica 21, 1, 39-60.

KhUlLER, S. 1995. Approximation algorithms for for finding highly connected subgraphs. In Approximation Algorithms for NP-hard problems, Chapter 6, D. S. Hochbaum, Ed. PWS, Boston, MA, USA, 236-265.

Kortsarz, G., Krauthgamer, R., and Lee, J. R. 2004. Hardness of approximation for vertexconnectivity network design problems. SIAM J. on Computing 33, 3, 704-720.

Kortsarz, G. AND Nutov, Z. 2007. Approximating minimum-cost connectivity problems. In Handbook of Approximation Algorithms and Metaheuristics, Chapter 58, T. F. Gonzalez, Ed. Chapman \& Hall/CRC.

Kortsarz, G. AND Nutov, Z. 2008. Tight approximation algorithm for connectivity augmentation problems. J. of Computer and System Sciences 64, 5, 662-670.

Lando, Y. And Nutov, Z. 2009. Inapproximability of survivable networks. Theoretical Computer Science 410, 21-23, 2122-2125.

MADER, W. 1978. A reduction method for edge-connectivity in graphs. Annals of Discrete Math 3, $145-164$.

Nagamochi, H. And Ishi, T. 2003. On the minimum local-vertex-connectivity augmentation in graphs. Discrete Applied Mathematics 129, 2-3, 475-486.

Nutov, Z. 2005. Approximating connectivity augmentation problems. In SODA. 176-185.

Nutov, Z. 2006. Approximating rooted connectivity augmentation problems. Algorithmica 44, $213-231$.

Nutov, Z. 2008. Approximating node connectivity augmentation problems. Manuscript. To appear in APPROX 2009.

PeleG, D. 2007. Approximation algorithms for the Label-CoverMAX and red-blue set-cover problems. J. of Discrete Algorithms 5, 1, 55-64.

RAZ, R. 1998. A parallel repitition theorem. SIAM J. on Computing 27, 3, 763-803.

Raz, R. And Safra, S. 1997. A sub-constant error-probability low-degree test and a sub-constant error-probability PCP characterization of NP. In STOC. 475-484.

Szigeti, Z. 1999. Hypergraph connectivity augmentation. Math. Programming 84, 519-527. 